\title{
COMPARISON OF PRE-OPERATIVE PERITONEAL WASH AND DRAINAGE VERSUS URGENT LAPAROTOMY STRATEGY IN PATIENTS WITH LATE STAGE PERFORATION PERITONITIS. A RANDOMIZED CONTROLLED TRIAL
}

\section{Salah Kadhim Muslim}

MB,ChB, CABS, FICMS, Consultant Surgeon, Basrah General Hospital. Assistant Professor of Surgery, Department of Surgery, College of Medicine, University of Basrah, IRAQ.

\begin{abstract}
Peritonitis is inflammation of peritoneum which is most commonly due to generalized or localized infection. Secondary peritonitis is the commonest form and a large percent of them is due to perforation or impending gastrointestinal perforation. The prognosis depends on multiple factors by which Mannheim Peritonitis Index (MPI) appears to be more practical.

This study aimed to assess the value of pre-operative aspiration of peritoneal fluid followed by peritoneal wash and drainage before proceeding to definitive surgery in patients with MPI score $>20$.

This prospective study was conducted in Al-Hussein Teaching Hospital in Al Nasserya city and Basrah Teaching Hospital in Basrah city from October 2003 to July 2014. Each patient admitted to the emergency department in these hospitals with the provisional diagnosis of perforation peritonitis was evaluated with MPI score. If the score was less than 20; the patient managed with resuscitation and broad spectrum antibiotics for 2-3 hours then by definitive surgery while those with MPI more than 20 were randomly divided into two groups; the first were managed with 2-3 hours resuscitation with intra-venous fluid resuscitation and antibiotics followed by urgent surgical exploration (USE). The second group were managed with percutaneous peritoneal drainage (PPD) with aspiration of the fluid and then irrigation of the peritoneal cavity with isotonic saline and followed by a drainage with aid of the gravity through another catheter located in the right ileac fossa.

Sixty two patients included in this study who fulfilled the criteria of perforation peritonitis and MPI score more than 20. Around half of them the score was between 26-30. Perforated peptic ulcer is the commonest etiology. The most common cause of their high MPI score is the late presentation and the evidence of organ failure. Renal failure is the most prevalent organ failed in both groups. The overall mortality is decreased in (PPD) group. Those with urgent surgical exploration(USE) showed no improvements in the pre-operative vital signs, prolonged operation time and a higher mortality and more severe post-operative complication than PPD group.

In conclusion, the pre-operative percutaneous peritoneal aspiration of the fluid followed by peritoneal irrigation and drainage in patients with advanced stage perforation peritonitis is associated with a significant improvement in the pre-operative pulse rate and blood pressure, decrease in the operation time, a decrease in the overall mortality and deep seated wound infection and dehiscence but it is associated with a higher mortality in the first post-operative day.
\end{abstract}

\section{Introduction}

Peritonitis is inflammation of
peritoneum which is most commonly due to generalized or localized infection. Secondary peritonitis is the commonest form $^{1,2}$. A large percentage of secondary peritonitis is due to perforation which if not treated surgically causes considerable mortality. Mortality of perforation peritonitis was as high as $90 \%$ in the early twentieth century and is still high despite advances in antibiotics, surgical technique, radiographic imaging, and 
resuscitation therapy. The contamination with the peritoneal cavity can lead to a cascade of infection, sepsis, multi system-organ failure (MSOF) and death if not treated in a timely manner ${ }^{3,4}$.

Many scoring systems was used to assess the severity of perforation peritonitis like Acute Physiology And Chronic Health Evaluation score(APACHEII), Simplified Acute Physiology Score (SAPS). In 1983; Wacha and Linder developed a scoring system consist of twenty risk factors on which eight of them proved to be of prognostic relevance. This system called Mannheim Peritonitis Index (MPI) (Table I) ${ }^{4,5}$. It appears to be more practical than other scoring systems. Mortality increases with increasing range of the score, adopting three cut-off ranges from less than 20, from 20 to 30 and more than 30 growing the mortality from $0 \%$ to $28 \%$ up to $81 \%$ respectively ${ }^{1,4,5}$.

\section{Table I: The Mannheim peritonitis index}

\begin{tabular}{|lc|}
\multicolumn{1}{l}{ Risk factor } & score \\
\hline Age $>$ 50 years & 5 \\
\hline Female sex & 5 \\
\hline Organ failure* & 7 \\
\hline Malignancy & 4 \\
\hline Preoperative duration of peritonitis $>24$ h 4 \\
\hline Origin of sepsis not colonic & 4 \\
\hline Diffuse generalized peritonitis & 6 \\
\hline Exudate & \\
\hline Clear & 0 \\
\hline Cloudy, purulent & 6 \\
\hline Fecal & 12 \\
\hline
\end{tabular}

*Kidney failure; creatinine level $>177$ umol/L or urea level $>167 \mathrm{mmol} / \mathrm{L}$ or oliguria $<20 \mathrm{ml} /$ hour. CardioPulmonary insufficiency; $\mathrm{PO} 2<50 \mathrm{mmHg}$ or PCO2 $>50 \mathrm{mmHg}$. Intestinal failure; obstruction/paralysis $>24$ hours or complete mechanical ileus. Shock hypodynamic or hyperdynamic. CNS failure; altered mental status. Hepatic failure; hyperbilirobinemia. Bone marrow failure; pancytopenia, coagulopathy.

In order to decrease the mortality and morbidity of a patient with perforation peritonitis, adequate fluid resuscitation should be started, initiation of antibiotics and then source control in the form of emergency surgery. The principles of source control dictate direct control of the site of perforation, evacuation of intra-peritoneal contamination, drainage of abscesses, debridement of necrotic tissue and foreign matter, and reestablishment of functional anatomy ${ }^{6}$. If there is delay in the initial management; many of patients developed systemic inflammatory response syndrome(SIRS), sepsis and septic shock with accompanying hemodynamic compromise, hypothermia, acidosis, and a coagulopathy. The challenge is how to manage such patients without causing further physiologic compromise. So, source control should be achieved with the least invasive maneuver ${ }^{7-9}$.

Sepsis source control through percutaneous peritoneal drainage is not new. Atakent YS et al and Lessin MS et al and others described a definitive treatment for acute intestinal perforation in extremely very low birth neonate by percutaneous peritoneal drainage ${ }^{10-12}$. Ramaswamy and Niall et al discuss the same principle in the treatment of bladder perforation during transurethral resection of prostate ${ }^{13}$. S.Peterson-Brown and H.A.F Dudly stated that "in a circumstances of severe abdominal distension, peritoneal aspirate should be considered by peritoneal dialysis catheter in order to permit the escape of gas and exudate, thereby providing an initial 
guide to the diagnosis in addition to relieving the cardiopulmonary effect of distension". They also stated that "if the patient's condition still prevents operation, treatment may began by running a liter of normal saline with or without antibiotics over a period of an hour and then recovering it by gravity drainage" ${ }^{14}$.

The aim of this study is to assess whether pre-operative drainage of purulent peritoneal fluid followed by peritoneal wash and drainage can decrease the mortality and the morbidity of patients with perforation peritonitis who had MPI score more than 20 .

\section{Patients and methods}

This prospective study was conducted in Al-Hussein Teaching Hospital in Al Nasserya city and Basrah General Hospital in Basrah city from October 2003 to July 2014. Each patient admitted in the emergency department of these hospitals with the provisional diagnosis of perforation peritonitis was re-evaluated by the resident surgeon on-call and then by the same specialist surgeon who scored each patient according to MPI index. Patients with peritonitis secondary to esophageal perforation and reproductive tract perforation and those who had acute abdominal pain of uncertain etiology were excluded from the study. Each patient with MPI index less than 20 were managed by rapid fluid resuscitation, broad spectrum antibiotics for 2-3 hours and then definitive surgery while those with index more than 20 were randomly divided into two groups of management; those presented on odd numbered days were managed with resuscitation followed by urgent surgical exploration (USE) and those who presented on even days were managed with percutaneous peritoneal drainage (PPD) which is performed by the following steps: 1- Informed verbal consent taken from the patient's family with full details about the procedure.
2- The patient was kept in supine position with painting the skin around the umbilicus followed by draping then, a 510 milliliters of $1 \%$ xylocaine as a local anesthesia was infiltered subcutaneously then a one centimeter incision in the skin just above the umbilicus followed by insertion of a 16 or $18 \mathrm{G}$ peritoneal dialysis catheter directed toward the pelvis, aspiration of the fluid(if any) found in the peritoneal cavity in $10 \mathrm{ml}$ syringe. The fluid drained was inspected for the color and sent for Gram stain and culture. Another tube drain (14 or 16 G)was inserted through a separate incision in right ileac fossa under local anesthesia as a pelvic drain. 3- A 1000 $\mathrm{ml}$ of warm isotonic saline was infused into the peritoneal cavity through peritoneal dialysis catheter with occlusion of pelvic drain for 30 minutes. Then release the occlusion and drain the wash through the pelvic tube drain with changing the patients' position to more head up position. This step was repeated each 3-4 hours for 24 hours. During that, patient's response was observed with a follow up chart in the ward or in ICU focusing on the conscious level, vital signs, urine output and oxygen saturation by pulse oxymetry. After that, the abdomen was then explored for definite procedure by the same surgeon.

Data were recorded including patients age, sex, pre-operative vital signs, the color, and the culture of peritoneal aspirate, the definitive diagnosis after surgical exploration, the duration of definitive surgical procedure and postoperative morbidity and mortality. Chisquare $(\chi 2)$ test was used to compare variables and tests were considered significant when P-Value $\leq 0.05$.

\section{Results}

Sixty two patient presented to the emergency department with a definitive diagnosis of perforation peritonitis and MPI index more than 20. They were 38 males and 24 females. Their age ranged 
between 14-90 years with a mean age of 39.7 years. They were randomly classified into two groups; percutaneous peritoneal drainage (PPD N:32) and urgent surgical exploration (USE N:30) as shown in table II.

Table II: gender distribution of the patients in the two groups.

\begin{tabular}{|l|l|l|l|}
\hline \multicolumn{2}{|l|}{$\begin{array}{l}\text { Percutaneous peritoneal drainage } \\
\text { PPD (N:32) }\end{array}$} & \multicolumn{2}{l|}{ urgent surgical exploration } \\
USE (N:30)
\end{tabular}

Late patients presentation to the surgical emergency department or to the surgeon, ranging between 4-17 days as shown in figure 1 .

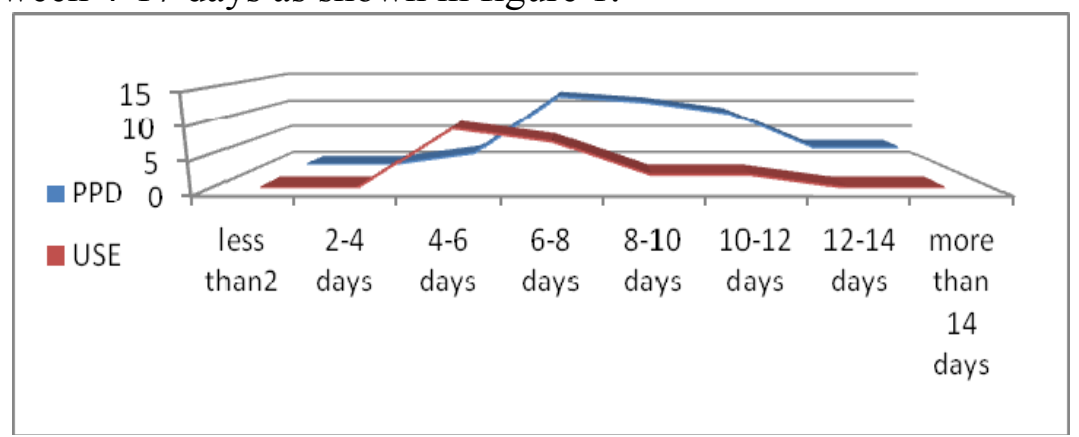

Figure:1: Time of first presentation to the surgical emergency department in days in both groups.

In both groups, the patients were further sub-classified into three groups according to MPI score (figure 2). It shows that around half of the patients presented with MPI between (26-30). Each patient was evaluated for evidence of organ failure at times of presentation which was discovered in $58(93.5 \%)$ patients.

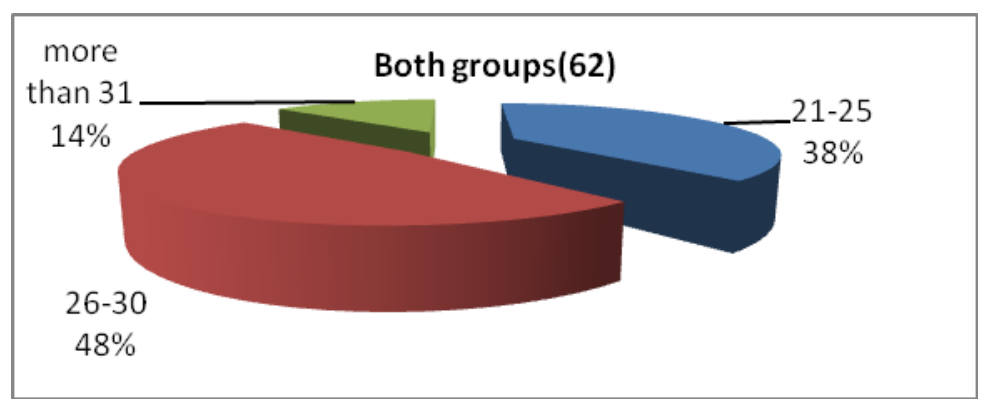

Figure 2: The MPI score in both groups.

Table III, shows the site of organ failure associated with both groups. Renal failure is the most prevalent organ failed in both groups $(50 \%)$. There was no patient who had a features of bone marrow failure or hepatic failure.

Table III: Organ failure in both groups.

\begin{tabular}{|l|l|l|l|l|}
\hline Organ failure & All (58) & USE (28) & PPD(30) & Percentage \\
\hline Renal impairment & 31 & 10 & 21 & $50 \%$ \\
\hline Cardio-pulmonary failure & 18 & 9 & 9 & $29 \%$ \\
\hline Intestinal failure & 15 & 9 & 6 & $24 \%$ \\
\hline CNS failure & 3 & 3 & 0 & $4.8 \%$ \\
\hline Bone marrow failure & 0 & 0 & 0 & $0 \%$ \\
\hline Hepatic failure & 0 & 0 & 0 & $0 \%$ \\
\hline
\end{tabular}


Multiple organ failure seen in six patients of PPD group and in three patient of USE group. All patients with CNS failure were those with MPI score more than 30 and associated with other organ failure.

Pre-operatively, a sample from the peritoneal fluid could be aspirated in $49(79 \%)$ patients which was then inspected for the color (figure 3).

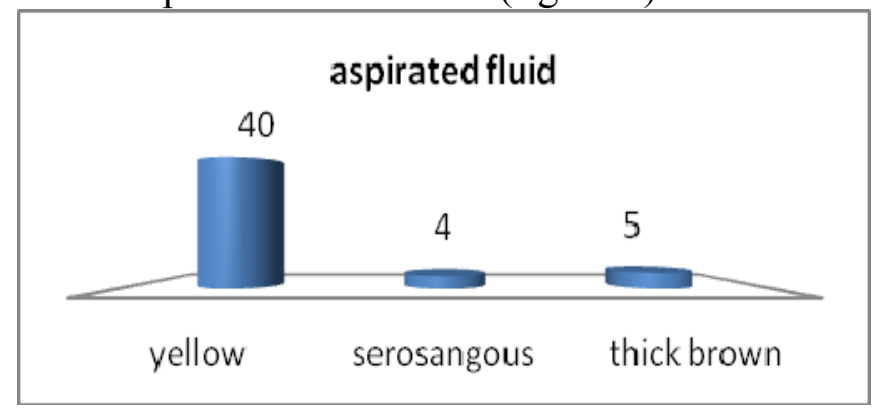

Figure 3: The color of the aspirated fluid.

In all patients, and on surgical exploration, a sample from the peritoneal fluid was aspirated and then sent for culture as shown in figure 4.

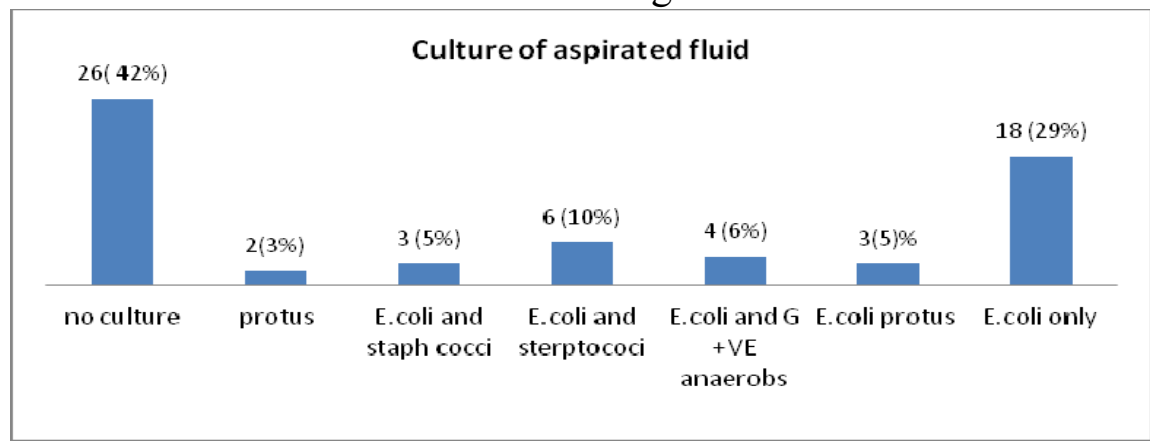

Figure 4: The result of aspirated peritoneal fluid culture .

It is clear that the most common bacteria cultured from the aspirate was E.coli with or without other enteric bacteria. Negative culture was detected in $26(42 \%)$ patients. The changes in the vital signs were evaluated on admission and then checked just before surgical interference in both groups as described in Table IV.

Table IV: The changes in mean vital signs on admission and just before operation in both groups.

\begin{tabular}{|c|c|c|c|c|c|c|}
\hline Parameter & $\begin{array}{l}\text { (USE N:30) } \\
\text { On admission }\end{array}$ & $\begin{array}{l}\text { pre- } \\
\text { operative }\end{array}$ & $\begin{array}{l}\mathrm{p} \text {. } \\
\text { value }\end{array}$ & $\begin{array}{l}\text { (PPD N:32) } \\
\text { On admission }\end{array}$ & $\begin{array}{l}\text { pre- } \\
\text { operative }\end{array}$ & $\begin{array}{l}\text { p. } \\
\text { value }\end{array}$ \\
\hline $\begin{array}{l}\text { Pulse ratel } \\
\text { minute }\end{array}$ & $\begin{array}{c}80-124 \\
(114)\end{array}$ & $\begin{array}{l}82-120 \\
(108)\end{array}$ & 0 & $\begin{array}{l}84-130 \\
(110)\end{array}$ & $\begin{array}{l}90-102 \\
(94)\end{array}$ & $*$ \\
\hline $\begin{array}{l}\text { Respiratory } \\
\text { ratelminute }\end{array}$ & $\begin{array}{l}20-24 \\
(21)\end{array}$ & $\begin{array}{l}20-24 \\
(22)\end{array}$ & o & $\begin{array}{l}26-30 \\
(27)\end{array}$ & $\begin{array}{c}25-30 \\
(27)\end{array}$ & o \\
\hline $\begin{array}{l}\text { Temperature } \\
{ }^{\circ} \mathrm{C}\end{array}$ & $\begin{array}{l}37.8-38.4 \\
(37.9)\end{array}$ & $\begin{array}{l}37.8- \\
38.5 \\
(38.1)\end{array}$ & 0 & $\begin{array}{l}37.5-38.2 \\
(37.9)\end{array}$ & $\begin{array}{l}37.5- \\
38.3 \\
(38.1)\end{array}$ & 0 \\
\hline $\begin{array}{l}\text { Sys. Blood } \\
\text { pressure }\end{array}$ & $\begin{array}{l}60-110 \\
(85)\end{array}$ & $\begin{array}{l}75-110 \\
(90)\end{array}$ & o & $\begin{array}{l}65-100 \\
(85)\end{array}$ & $\begin{array}{l}80-110 \\
(105)\end{array}$ & $*$ \\
\hline
\end{tabular}


There were statistical significant decline in the pulse rate and increase in the blood pressure in PPD group only.

In both groups, an intravenous anti-biotic cover was started on admission either triple antibiotics (ampicillin QDS, gentamycin TDS, metronidazole TDS) or third generation cephalosporin BID with metronidazole. The patients were finally explored through midline laparotomy for definitive diagnosis and management. Figure 5 shows the underlying causes of perforated peritonitis in all patients.

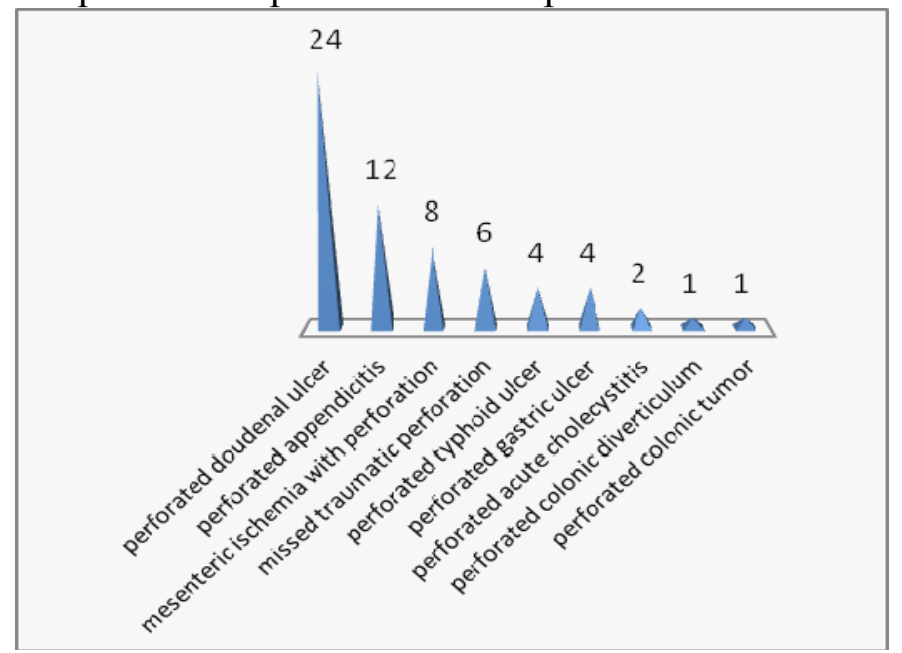

Figure 5: the definitive diagnosis in both groups .

The average time of the whole surgical procedure was calculated in both groups. In PPD group it ranged between 50-130 minutes with a mean time 61.2 minutes. In USE group it ranged between 50-120 minutes with a mean time 83 minutes which shows a statistical significant reduction in operative time in PPD group as shown in figure 6.

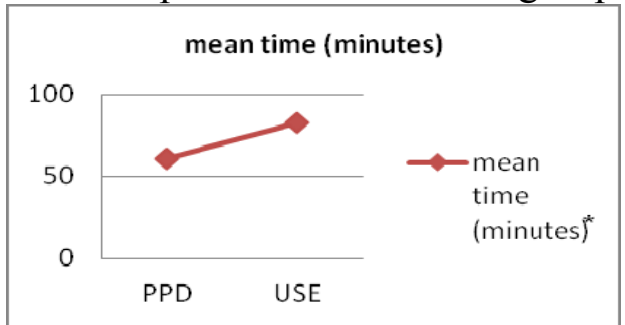

Figure 6: The differences in the meantime of surgery between the two groups.

Overall mortality in both groups was $35.4 \%$ (22 patients):40\% (12) in USE group and $31.2 \%$ (10) in PPD group. This differences is statistically significant. Figure7 shows the percent mortality in association with time of death. Most of deaths $(70 \%)$ in PPD group were occurred in the first 24 hours. In USE group; although the highest mortality was in the first 24 hours postoperatively, it is clear that the patients still had a significant mortality after that with a maximal incidence in the second post operative day.

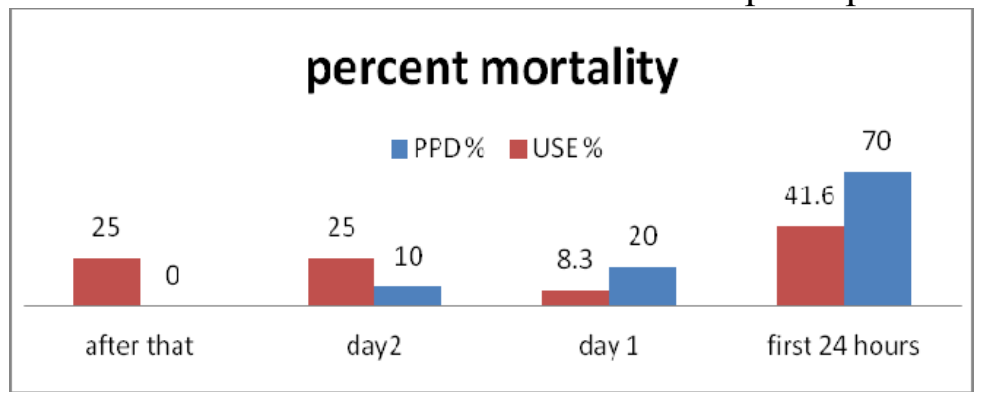

Figure 7: The percent mortality in the post-operative days. 
All patients in both groups who died in the first 24 hours after surgery show no recovery from anesthesia and they continue on mechanical ventilation after surgery till development of a cardiac arrest and death. In PPD group; those who survive the first 24 hours showed a significant decrease in mortality which was mostly due to a complication of organ failure; while those in USE group showed persistent risk of death from a complication of organ failure and sepsis from residual septic foci as shown in table V.

Table V: The cause of death in both groups in the post-operative period

\begin{tabular}{|l|l|l|l|}
\hline DAYS & USE(12) & PPD(10) & Possible causes \\
\hline Day 0 & 5 & 7 & Anesthetic ( on mechanical ventilation) \\
\hline Day 1 & 1 & 2 & Cardiac arrhythmia + ischemia \\
\hline Day 2 & 2 & 1 & Suspected pulmonary embolism \\
\hline & 1 & & Acute renal failure + anuria \\
\hline$>2$ days & 1 & 0 & Burst abdomen ( re-exploration) \\
\hline & 1 & & High output entero-cutanous fistula \\
\hline & 1 & & Septic shock(multiple residual abdominal abscesses) \\
\hline
\end{tabular}

After recovery from anesthesia, all patients were kept on a closed and a regular observation chart for any evidence of surgical complications which is seen 12 patients in each group. Table VI shows the surgical complications observed in both groups.

Table VI: Postoperative morbidity in both groups. * * (statistical significance)

\begin{tabular}{|l|lc|l|l|}
\hline Surgical complications & \multicolumn{2}{|l|}{ USE 12 patients (40\%) } & PPD = 12 patients (37.5\%) \\
\hline Superficial wound infection & 2 & $(16.7)^{*}$ & 5 & $(41.6)^{*}$ \\
\hline Deep wound infection & 7 & $(58.3)^{*}$ & 4 & $(33.3)^{*}$ \\
\hline Burst abdomen & 2 & $(16.7)^{*}$ & 1 & $(8.3)^{*}$ \\
\hline Entero-cutaneous fistula & 1 & $(8.3)$ & 1 & $(8.3)$ \\
\hline Chest infection & 0 & $(0)$ & 1 & $(8.3)$ \\
\hline
\end{tabular}

It is clear that $40 \%$ of patients in USE had postoperative complications compared to $37.5 \%$ in PPD group. In addition, the latter group showed a higher rate of superficial surgical site infection and a lower rate of deep seated wound infection and dehiscence than USE group.

On comparing the percent mortality in association with MPI score; it is found that there is no statistical significant differences between the two groups as in figure 8 .

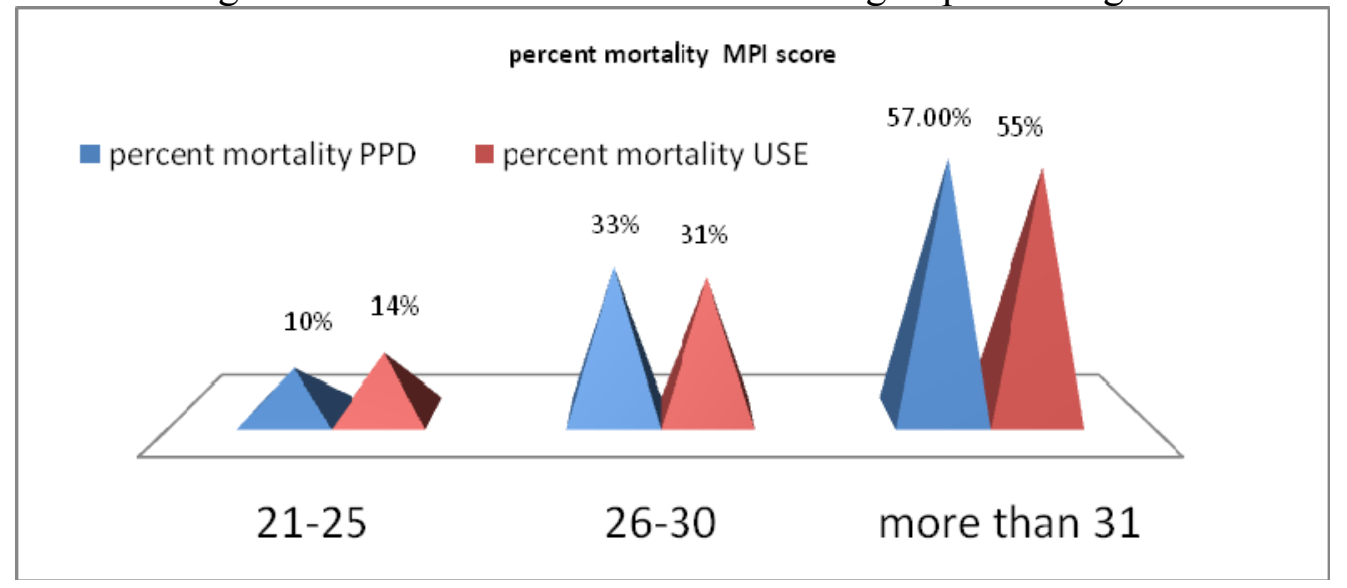

Figure 8: Percentage of mortality in MPI score in both groups. 


\section{Discussion}

In the first quarter of the twentieth century; Martin Kirschner first described the standard surgical approach for treatment of perforation peritonitis ${ }^{15}$. It consist of source control, reduction in bacterial contamination and prevention of its recurrence ${ }^{7}$. Other measures are of little use if the operation does not successfully abort the infective source and quantitatively reduce the inoculation of micro-organisms and adjuvant of infection so that they can be effectively handled by the patient's defenses, supported by antibiotic therapy, ${ }^{7,15-18}$

In this study, patients with advanced peritonitis were selected depending on their high MPI index. The cause of which is mainly due to the delay in presentation and evidence of organ failure. There are multiple factors explain such delay; either the patients ignored the earlier symptoms, or had taken herbal or medicinal care by local health facility, or those patients lived in places far from centers with surgical facilities, so had to travel long distances to reach a referral center. Such delay leads to widespread dissemination of the primary insult making the control of pathology difficult and resulting in poor intraoperative outcome. It could be regarded as the most important factor predicting in the prognosis of peritonitis especially perforation peritonitis ${ }^{6,12}$.

In this study, renal system is the most common organ failed followed by cardiopulmonary system. These findings are different from other reports like that reported by Ali Yaghoobi etal and Betra who found that the most common organ failed is cardiopulmonary followed by the hepatic failure ${ }^{4,5}$. Exact explanation for such differences is unknown. Gastro-intestinal failure lacked clear definition, its incidence was low and its occurrence was rarely associated with poor outcome. This study reported also that failing central nervous system is associated with other organ failure, high MPI score and a significant mortality ${ }^{4}$.

Although E.coli and other Gram -ve enterococci are considered the most common intra-abdominal pathogens, the data presented here indicate that these are the GIT flora and much more causing peritonitis when spilled to the peritoneal cavity converting into pathogenic one ${ }^{19}$. Also because these micro-organism are more prone to determine septicemia for their high affinity to reach the blood circulation. Anaerobic and opportunistic bacteria usually leads to circumscribed peritonitis ${ }^{1}$.

Negative cultures were found in around one third of our patients; a finding which is similar to others ${ }^{4,5}$ and can be explained either by the chemical rather than bacterial nature of peritonitis that occurs specially with upper GIT perforation before proceeding to a secondary bacterial peritonitis or due to the effect of pre-operative use of antibiotics $^{20}$.

During pre-operative resuscitation in both groups; a statistically significant change in both pulse rate and in systolic blood pressure were observed in PPD group while no significant improvement was reported in the temperature and respiratory rate. These findings although were not reported by the others; but clearly indicates that the 24 hours of resuscitation elapsed between the diagnosis of perforation peritonitis and the definitive source control was enough to make an apparent improvement in the pulse rate and systolic blood pressure but it was an inadequate time for the thermo-regulatory and acid-base balance which needs more time to show a significant changes. In addition; the cause of tachypnea which is mainly due to lactic acidosis and the source of hyperthermia which is the pyrogens and the inflammatory mediators released from the inflammatory cells which is not eliminated during that time ${ }^{19}$.

On surgical exploration, the commonest underlying etiology is perforated peptic ulcer followed by perforated acute appendicitis. This finding is consistent with other researches in the nearby countries ${ }^{21}$ while different from other researches done in 
Europe, USA and Japan in which acute perforated appendicitis is the most common cause followed by colonic perforation mostly due acute diverticulitis ${ }^{4,5,19,20,22}$. There is a significant drops in the incidence of proximal bowel perforation in the developed nations due to the better availability of proton pump and adoption of therapies against Helicobacter pylori. Distal bowel perforations especially colonic perforations are the leading cause of perforation peritonitis in the western world ${ }^{2,23,24}$.

Regarding the time needed to complete the surgical procedure, there is a statistically significant decrease in the time in PPD group in which there is less time of initial suction of peritoneal fluid and less peritoneal wash after completion the source control. This is because that some of these actions were already started pre-operatively.

There is a wide range of in-hospital mortality of patients with perforation peritonitis $(3 \%-58 \%)^{4,18,22,23}$, but the mortality rate among patients with high severity scores is ranging between $(34-58 \%)^{23-28}$. This study results are consistent with this range in both groups but there is a statistical significant decrease in overall mortality in PPD group. in the first 24 hours after surgical exploration, the mortality rate of PPD group is more than USE group in which the patients did not recover from anesthesia. This finding may be explained by the prolonged effect of the myocardial depressant factors like tumor necrotic factor-alfa (TNF-alpha), platelet activating factor (PAF), gamma interferon (IFN-gamma) and arachidonic acid metabolites which can continue for days and further depressing cardiac function even with adequate intravenous fluid therapy keeping in mind that before anesthesia; sufficient preoperative systemic blood pressure is not indicative of adequate volume status, and therefore, heart rate, urine output, and mental status should all be considered when evaluating the volume ${ }^{7}$. Additionally, there is a risk although theoretical that the increase intra-abdominal pressure created by both pre-operative peritoneal lavage and the positive pulmonary ventilation during anesthesia will increase the opening of special holes found in the peritoneal surface of the diaphragm called lymphatic lacunae through which bacteria and toxins can pass directly to the blood stream through the thoracic duct. This complication can be minimized by frequent suctioning of peritoneal fluid with the least volume of peritoneal lavage.

While peritoneal lavage for peritonitis is universally recommended, remarkably little studies has been done on its specifics. Too many articles on peritoneal lavage in peritonitis are available but only one small clinical control trial by Schein in 1990, comparing no lavage with intra-operative lavage with and without antibiotics, showed no differences in survival. As a result, Schein recommends only "swabbing or mopping peritoneal surfaces with moist laparotomy packs"28-30. Despite this, intra-operative lavage remains standard therapy. It is recommended that all fluid be aspirated at the closure of the abdomen as there is evidence that the ongoing presence of fluid decreases macrophage effectiveness. Studies looking at post-operative continuous lavage showed limited success with increasing risk of fistulization ${ }^{7,29}$. The latter surgical complication was also associated with radical debridement of fibrinous exudates. One trial showed it to be more dangerous than lavage alone and it has been abandoned. Addition of antibiotics to lavage solution also has not been shown valuable, neither has postoperative continuous lavage or use of drains ${ }^{18}$.

Post- operatively, both groups showed no statistical significant differences in surgical complications in general, but USE group showed a statistically significant higher incidence of deep seated wound infection and intra-abdominal abscess and hence burst abdomen than PPD group. This finding is explained by the prolonged reduction of bacterial contamination in PPD group which was started before definitive surgical repair during which the time was spent for only opening the contaminated spaces, aspirating 
the remaining purulent fluid and removing food, feces and foreign debris while in USE group all these actions should be done in limited time in addition to peritoneal lavage.

Conclusions, the pre-operative percutaneous peritoneal aspiration of the fluid and peritoneal irrigation and drainage in patients with advanced stage of perforation peritonitis documented by their high MPI score for 24 hours before definitive surgical source control is associated with a significant improvement in the pre-operative pulse rate and blood pressure, decrease in the operation time, a decrease in the overall mortality and deep seated wound infection and dehiscence but it is associated with a higher mortality in the first postoperative day indicating that the 24 hours of preoperative wash and drainage is not enough to eliminate all the factors that are responsible for increasing mortality.

\section{References}

1- Anderea Cavallaro,Vito Cataneo . Management of secondary peritonitis . Our experience. Ann .Ital. Chir.2008;79:255-260.

2- Jeetendar J Paryani, Vikas Patel, Gunvant Rathod. Etiology of peritonitis and factors predicting the mortality in peritonitis.. National Journal of Community Medicine ;Volume 4, Issue 1, Jan-Mar 2013:145.

3- John T. Langell, Sean J. Mulvihill. Gastrointestinal Perforation and the Acute Abdomen. Medical Clinic of North America 92 (2008): 599-625.

4- Ali Yaghoobi Notash, Javad salami . Evaluatin of Mannheim peritonitis index and multiple organ failure score in patients with peritonitis . Indian journal of gastroenterology 2005; Vol. 24 September-October:197-204.

5-Batra P., Gupta D., Batra R., Kothari R. and Deshmukh P.R. Mannheim peritonitis index as an evaluative tool in predicting mortality in patients of perforation peritonitis. CIB Tech Journal of Surgery 2013; Vol. 2(3) September-December: 30-36

6- Basnet RB, Sharma VK. Evaluation of predictive power of Mannheim Peritonitis Index .Postgraduate medical journal of NAMS; Volume 10.Number 2 Jul-Dec 2010: 10-13.

7- Amir Baluch, Asan Janoo, Kim Lam, Jason Hoover and Alan Kaye. Septic shock: Review and anesthetic considerations; M.E.J ANESTH. $19(1) ; 2007: 72$

8- Brenda Brancheau, RN. Anesthesia considerations for the patient with septic shock. Journal of the American Association of Nurse Anesthetists; October $1984: 549-552$

9- C. M. Lamb, P. MacGoey, A. P. Navarro and A. J. Brooks. Damage control surgery in the era of damage control resuscitation. British Journal of Anesthesia (2014); 113 (2): 242-9.

10-Atakent YS1, Wasserman-Hoff R, Ozek E, Oygur N, Ginsburg HB. Percutaneous peritoneal drainage in the management of acute intestinal perforation. J Perinatol. 1997 Jan-Feb;17(1):46-51.

11- Lessin MS1, Luks FI, Wesselhoeft CW Jr, Gilchrist BF, Iannitti D, DeLuca FG. Peritoneal drainage as definitive treatment for intestinal perforation in infants with extremely low birth weight (<750 g). J Pediatr Surg. 1998 Feb;33(2):370-372.

12- Supreethi Kohli, Anu Singhal, Anita Arora. Spontaneous Biliary Peritonitis in Children. Journal of Clinical Imaging Science ;Vol. (3). Issue 2 Apr-Jun 2013: 1-4

13- Ramaswamy Manikandan, Niall Lynch, and Richard J. Grills .Percutaneous Peritoneal Drainage for Intraperitoneal Bladder Perforations during Transurethral Resection of Bladder Tumors. Journal of Endourology. December 2003; 17(10): 945-947.

14- S. Paterson Brown, H.A.F.Dudley and B.W.Ellis. Hamilton Bailys Emergency Surgery. Twelfth Edition. Butterworth Heinemann Ltd . International edition 1995. Chapter 26. Intraperitoneal sepsis p335.

15- Schoin M,Saadia R, Froinkel Z, Decker GAG. Aggressive treatment of severe diffuse peritonitis from intestinal origin. World .J. Surg 1990;14:210-7.

16- H.M.Radford. Sepsis, sever sepsis and septic shock in adults and anesthesia. South African journal and analgesia. May2002:30

17- Dietmar H. Wittmann, Moshe Schein, and Robert E. Condon. Management of Secondary Peritonitis. Annals of surgery. Vol. 224, No. 1: $10-18$.

18- Bosscha K, van Vroonhoven TJ, van der WC. Surgical management of severe secondary peritonitis. British Journal of Surgery 1999 ;86(11):1371-79.

19- Ashwini Kumar, Mark T. Muir, Stephen M. Cohn, Marc A. Salhanick, Daniel B. Lankford, and Venkata S. Katabathina. The etiology of pneumo-peritoneum in the 21st century. Acute Care Surgery. Volume 73, Number 3. p.542.

20- Alonzo P. Walker, Candace J. Krepel, Claudia M. Gohr and Charles E. Edmiston. Micro flora of Abdominal Sepsis by Locus of Infection. Journal of Clinical Microbiology, Feb. 1994, p. 557-558

21- Rajandeep Singh Bali, Sushant Verma, P. N. Agarwal ,Rajdeep Singh and Nikhil Talwar. Perforation Peritonitis and the Developing World. ISRN Surgery. Volume 2014 . p 1- 4.

22- Maurice King. Peritonitis and Intra-abdominal Abscess - Pelvic and Sub-phrenic. Primary Surgery. Volumes 1 \& 2. Oxford university press. $1990: 2-5$

23- Caroline C. Johnson, James Baldessarre, Matthew E. and R. W. Johnson. Peritonitis: Update on Pathophysiology, Clinical Manifestations, and Management. Clinical Infectious Diseases 1997;24:1035-47.

24- Hoch J. Stercoral peritonitis. Klinická Onkologie Zvláśtní Ėíslo 1999: 30-33.

25- Sanjay Gupta and Robin Kaushikn. Peritonitis - the Eastern experience. World Journal of Emergency Surgery 2006, 1:13p 1-6

26- Oddeke van Ruler; Cecilia W. Mahler; Kimberly R. Boer; et al. Comparison of On-Demand vs Planned Relaparotomy Strategy in Patients With Severe Peritonitis: A Randomized Trial. JAMA. 2007;298(8):865-872

27- Joseph S. Solomkin,1 John E. Mazuski. Diagnosis and Management of Complicated Intra-abdominal Infection in Adults and Children: Guidelines by the Surgical Infection Society and the Infectious Diseases Society of America • CID 2010:50 (15 January) .p 133

28- Dietmar H. Wittmann. Moshe Schein and Robert E. Condon. Management of Secondary Peritonitis .Annals of surgery .Vol. 224 , No.1. p $10-18$.

29- Yale D. Podnos, Juan Carlos Jimenez, and Samuel E. Wilson. Intra-abdominal Sepsis in Elderly Persons. Aging and infectious diseases. 2002:35 (1 July)

30- R. Phillip Dellinger, MD; Jean M. Carlet . Surviving Sepsis Campaign guidelines for management of severe sepsis and septic shock. Critical Care Med 2004 Vol. 32, No. 3. 